\title{
Wall shear stress and flow stability in a model fusiform aneurysm
}

\author{
G. J. Sheard ${ }^{1} \quad$ K. Ryan ${ }^{2}$
}

(Received 12 August 2008; revised 28 August 2008)

\begin{abstract}
Levels of wall shear stress in arterial blood flow are known to contribute to the integrity of wall tissue, and are believed to have a bearing on the pathogenesis of aneurysm disease. The flow of fluid through a model aneurysm was computed using a spectral element algorithm to study the distribution of wall shear stress and the stability of the flow to non-axisymmetric perturbations. Substantially higher mean and fluctuating wall shear stress components were found in the distal (downstream) region of the aneurysm bulge when compared to recent experimental measurements. A global stability analysis demonstrates that flow conditions consistent with a human aorta will produce nonaxisymmetric flow in an aneurysm consistent with that modeled here. These results demonstrate the necessity for detailed numerical modeling of these flows to accurately resolve the near wall fluid stresses.
\end{abstract}

See http://anziamj.austms.org.au/ojs/index.php/ANZIAMJ/article/view/ 1420 for this article, (c) Austral. Mathematical Soc. 2008. Published August 29, 2008. ISSN 1446-8735 


\section{Contents}

1 Introduction

C2

2 Problem formulation $\quad$ C4

2.1 Aneurysm model . . . . . . . . . . . . . . . . C C4

2.2 Governing equations . . . . . . . . . . . . C5

3 Numerical treatment C5

3.1 Numerical discretization . . . . . . . . . . . . C5

3.2 Pulsatile blood flow . . . . . . . . . . . . . C7

4 Results

C8

5 Conclusions

$\mathrm{C} 12$

References

C13

\section{Introduction}

Aneurysms are a form of arterial disease characterised by a localised weakening and dilation of an artery [9]. Factors that contribute to the weakening of the wall tissue include genetic predisposition [1], lifestyle [15], and irregular wall shear stress [7]. Ultimately, this weakening leads to rupture of the aneurysm. For aneurysms on the abdominal aorta, rupture is catastrophic [12], leading to death from internal bleeding in $75-90 \%$ of cases. The wall shear stress within an aneurysm is a function of the fluid mechanics of blood within the aneurysm. Therefore an understanding of the development of aneurysms requires an understanding of the blood flow dynamics within aneurysms, which forms the basis of this study.

Studies have identified that endothelial cells lining the interior of blood 


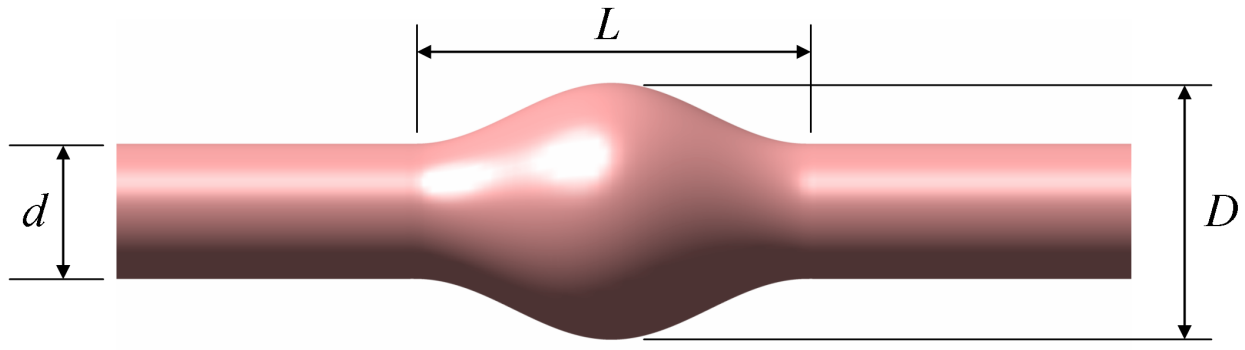

FiguRE 1: Schematic diagram of geometry under investigation, showing key dimensions. Fluid flows through the interior of the model.

vessel walls are susceptible to damage under excessive levels of wall shear stress [5], with a measured yield stress of $37.9 \pm 8.5 \mathrm{~Pa}$ reported. This value is almost an order of magnitude higher than the maximum wall shear stress measured in a healthy abdominal aorta (4.5 Pa [11]). Other studies [8] demonstrated a correlation between reduced fluctuation in wall shear stress and thickening of the innermost layer of the vessel wall. Hence the integrity of the wall of an artery can suffer from atypical wall shear stress environments featuring either excessive or inadequate stress levels.

Fusiform aneurysms are the focus of the present study, and manifest as a widening about the vessel centreline [9]. A recent experimental investigation attempted to systematically investigate aneurysm fluid dynamics through variation in geometric parameters [13]. In that study, velocity fields within in vitro aneurysm models were measured using particle image velocimetry (PIV). They reported smaller peak wall shear stresses within the aneurysm than in a healthy vessel. 


\section{Problem formulation}

\section{$2.1 \quad$ Aneurysm model}

The system under investigation comprises of a circular tube of diameter $\mathrm{d}$ featuring an axisymmetric bulge, and fluid flows through the tube driven by a time varying pressure gradient. The bulge is defined by a sinusoidal profile with length $L$ and maximum diameter $D$ (see Figure 1 ). Relative to a cylindrical $(z-r)$ coordinate system centered at the middle of the bulge, the tube wall and bulge are therefore defined by the function

$$
r^{\prime}\left(z^{\prime}\right)=\left\{\begin{array}{cl}
1 / 2 & \text { if }\left|z^{\prime}\right|>\mathrm{LR} / 2 \\
\frac{\mathrm{DR}+1}{4}+\frac{\mathrm{DR}-1}{4} \cos \left(\frac{2 \pi z^{\prime}}{\mathrm{LR}}\right) & \text { if }\left|z^{\prime}\right| \leq \mathrm{LR} / 2
\end{array}\right.
$$

where primes denote non-dimensionalisation by $\mathrm{d}$, and a diameter ratio $\mathrm{DR}=$ $\mathrm{D} / \mathrm{d}$ and length ratio $\mathrm{LR}=\mathrm{L} / \mathrm{d}$ are introduced.

Dynamic similarity of pulsatile Newtonian flow is achieved by matching two dimensionless parameters, the Reynolds number and Womersley number.

The Reynolds number is defined as $\operatorname{Re}=\mathrm{Ud} / v$, where $\mathrm{U}$ is the timeaveraged velocity in the tube, and $v$ is the kinematic viscosity. The timeaveraged velocity $\mathrm{U}=\overline{\mathrm{Q}} / \mathrm{A}$, where $\overline{\mathrm{Q}}$ is the time-average volume flow rate and $A$ is the tube cross-section area $\pi \mathrm{d}^{2} / 4$. The Womersley number is defined as $\alpha=(d / 2) \sqrt{2 \pi f / v}$, where $f$ is the frequency of the pulsatile waveform.

In a fluid flow, the local rate of rotation is obtained from the curl of the velocity field (the vorticity $\omega$ ). It can be shown that the zero tangential velocity gradient of the no-slip wall condition gives for the wall shear stress WSS $=\left.\mu \omega\right|_{\text {wall }}$, where $\mu$ is the dynamic viscosity. Here, vorticity and wall shear stress are non-dimensionalised by $\mathrm{U} / \mathrm{d}$ and $\mu \mathrm{U} / \mathrm{d}$, respectively.

Flow conditions $\operatorname{Re}=330$ and $\alpha=10.7$ were employed by Salsac et al. [13] to model conditions within a human abdominal aorta. The same Reynolds 
number and Womersley number are used throughout this study, and an aneurysm with dimensions $\mathrm{LR}=2.9$ and $\mathrm{DR}=1.9$ is considered, corresponding to Model 3 in that study.

\subsection{Governing equations}

To replicate the conditions of earlier experiments [13], blood is modeled as a Newtonian fluid. Axisymmetric flow is computed in cylindrical coordinates, governed by the incompressible Navier-Stokes equations

$$
\begin{aligned}
\frac{\partial u_{z}}{\partial t}+\left(u_{z} \frac{\partial u_{z}}{\partial z}+u_{r} \frac{\partial u_{z}}{\partial r}\right) & =-\frac{\partial P}{\partial z}+\frac{1}{\operatorname{Re}}\left[\frac{\partial^{2} u_{z}}{\partial z^{2}}+\frac{1}{r} \frac{\partial}{\partial r}\left(r \frac{\partial u_{z}}{\partial r}\right)\right] \\
\frac{\partial u_{r}}{\partial t}+\left(u_{z} \frac{\partial u_{r}}{\partial z}+u_{r} \frac{\partial u_{r}}{\partial r}\right) & =-\frac{\partial P}{\partial r}+\frac{1}{\operatorname{Re}}\left[\frac{\partial^{2} u_{r}}{\partial z^{2}}+\frac{1}{r} \frac{\partial}{\partial r}\left(r \frac{\partial u_{r}}{\partial r}\right)-\frac{u_{r}}{r^{2}}\right], \\
\frac{\partial u_{z}}{\partial z}+\frac{\partial u_{r}}{\partial r}+\frac{u_{r}}{r} & =0
\end{aligned}
$$

where the advection term is shown in convective form. The axial and radial velocity components are denoted by $u_{z}$ and $u_{r}$, respectively. The kinematic static pressure $\mathrm{P}$ is non-dimensionalised by $\mathrm{U}^{2}$, spatial coordinates by $\mathrm{d}$, and velocities by $\mathrm{U}$.

\section{Numerical treatment}

\subsection{Numerical discretization}

The flows in this study are computed by solving the time dependent incompressible Navier-Stokes equations in cylindrical coordinates using a spectral element method for spatial discretization of the flow domain, and a third order accurate backwards multistep time integration algorithm to evolve the 


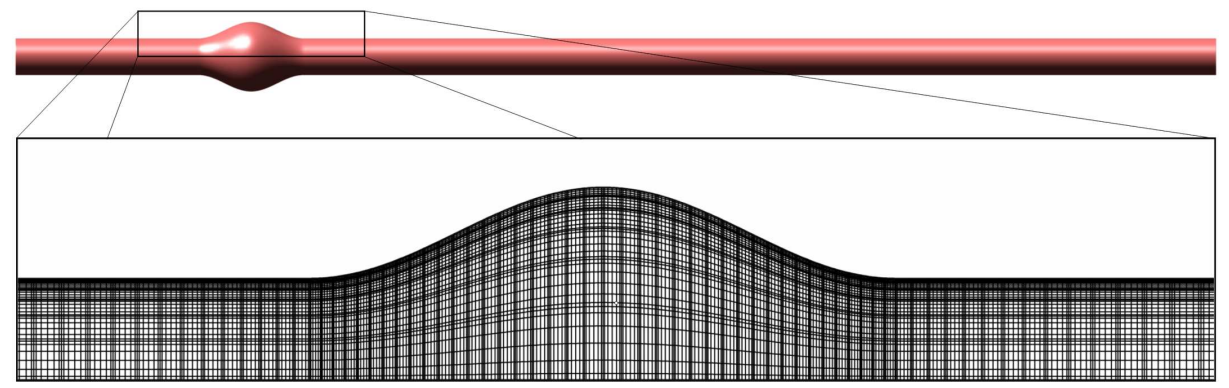

Figure 2: Top: The full computational domain. Inset: Detail of the mesh in the vicinity of the aneurysm bulge. Interpolation points corresponding to seventh-degree polynomials within each element are shown.

flow [6]. The present code has previously been validated and used to solve pressure driven flows in tubes [14].

On the axis, a stress-free boundary is naturally imposed through the formulation of the solver in cylindrical coordinates [4]. Along the tube wall, a no-slip condition is imposed on the velocity field, and an appropriate highorder condition is imposed on the pressure gradient normal to the wall [6]. The flow is driven by a pressure gradient, imposed using time varying Dirichlet conditions on pressure at the inlet and outlet, giving a pressure drop $\Delta \mathrm{P}=\mathrm{P}_{\text {out }}-\mathrm{P}_{\mathrm{in}}$. Periodic boundary conditions for velocity link the outlet to the inlet, $\mathbf{u}_{\text {out }}=\mathbf{u}_{\text {in }}$. To isolate the effect of a single aneurysm bulge, the computational domain included $30 \mathrm{~d}$ of straight tube, which was sufficient to dissipate the vortical structures generated within the bulge. The computational domain and spectral-element mesh are shown in Figure 2. The mesh contained 1099 nodes, forming 936 quadrilateral elements. Simulations used a zero velocity field as the initial condition, and were evolved to a periodic state before the data reported in this study was acquired.

A p-refinement study was conducted on a model with $\mathrm{LR}=2.9$ and $\mathrm{DR}=$ 1.9 at $\operatorname{Re}=330$ and $\alpha=10.7$. It was found that an element polynomial order 
of 7 provided discrepancies of less than $0.2 \%$ in flow rate when compared to a grid independent solution. This polynomial degree was used thereafter.

A global linear stability analysis was conducted using an implicitly restarted Arnoldi method to solve the eigenvalue problem [2, 3, e.g.] arising from the evolution of non-axisymmetric perturbation fields with azimuthal mode number $m$ on the periodic axisymmetric base flow. The Arnoldi solver was implemented using the ARPACK package [10].

\subsection{Pulsatile blood flow}

In 1955, Womersley [16] published an analytical solution for the time varying velocity profile and flow rate in a straight circular tube subject to a periodic pressure gradient. Adapting equation (21) from that study yields a relationship between the dimensionless Fourier coefficients of the present imposed kinematic static pressure drop $\left(\Delta \mathrm{P}_{\mathrm{k}}\right)$ and the mean velocity $\left(\overline{\mathrm{u}}_{\mathrm{k}}\right)$,

$$
\begin{aligned}
\Delta \mathrm{P}_{0} & =-\frac{32 \mathrm{~L}_{\text {tot }}}{\operatorname{Re}} \overline{\mathrm{u}}_{0}, \\
\Delta \mathrm{P}_{\mathrm{k}} & =i \frac{4 \mathrm{~L}_{\text {tot }}}{\operatorname{Re}} \frac{\alpha_{k}^{2}}{1-\frac{2}{\alpha_{k} i^{3 / 2}} \mathrm{~J}_{1}\left(\alpha_{k} i^{3 / 2}\right) / J_{0}\left(\alpha_{k} i^{3 / 2}\right)} \bar{u}_{k},
\end{aligned}
$$

where Bessel functions of the zeroth $\left(\mathrm{J}_{0}\right)$ and first $\left(\mathrm{J}_{1}\right)$ order with complex arguments are employed, $\mathrm{L}_{\text {tot }}$ is the dimensionless length of the computational domain (here $\mathrm{L}_{\mathrm{tot}}=32.9$ ), and $\alpha_{\mathrm{k}}=\sqrt{\mathrm{k}} \alpha$ characterises the frequency of the $k$ th mode. The appearance of the imaginary unit in these expressions demonstrates that the pressure drop and flow rate are out of phase, with the phase difference varying with the frequency of the waveform components $\left(\alpha_{k}\right)$.

While equations (1)-(2) are derived from a solution for a uniform straight tube, it was found that the aneurysm bulge did not significantly alter the pulse waveform. 
(a) $t=0.0 \mathrm{~T}$

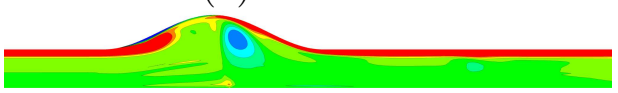

(c) $t=0.2 T$

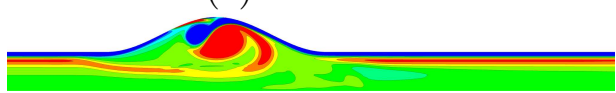

(e) $\mathrm{t}=0.4 \mathrm{~T}$

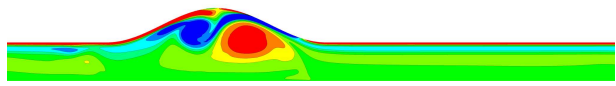

(g) $t=0.6 \mathrm{~T}$

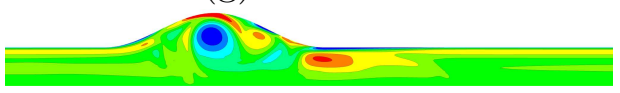

(i) $\mathrm{t}=0.8 \mathrm{~T}$ (b) $t=0.1 \mathrm{~T}$

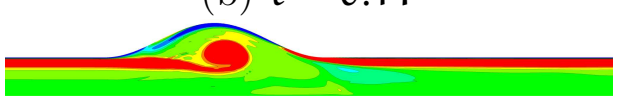

(d) $\mathrm{t}=0.3 \mathrm{~T}$

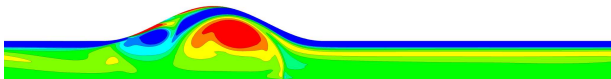

(f) $\mathrm{t}=0.5 \mathrm{~T}$

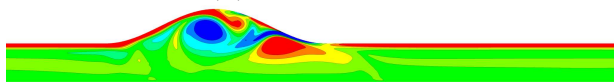

(h) $\mathrm{t}=0.7 \mathrm{~T}$

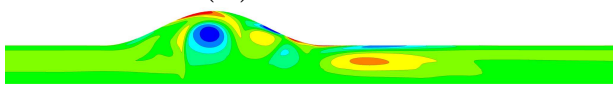

(j) $t=0.9 T$

\section{(}
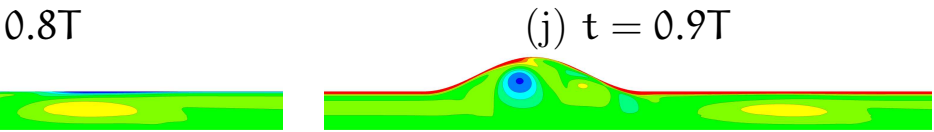

Figure 3: Out-of-plane vorticity contours at $R e=330$ and $\alpha=10.7$, with $\mathrm{DR}=1.9$ and $\mathrm{LR}=2.9$. Ten frames are shown over one period of the flow. Eight equi-spaced contour levels are plotted between $-20 \mathrm{U} / \mathrm{d}$ (blue) and $+20 \mathrm{u} / \mathrm{d}$ (red).

\section{Results}

Simulations were evolved to a periodic state subject to the imposed periodic pressure gradient, and data was acquired at a set of discrete intervals over a complete pulse cycle.

Figure 3 plots contours of vorticity over one period. Frame (a) shows the flow at peak systole (peak forward flow). As the flow rate begins to decrease, frame (b), particles roll up into a vortex located just downstream of the middle of the aneurysm. This vortex enlarges through frame (c), where its anti-clockwise rotation couples with reversed flow in the tube to generate 
a weaker vortex of opposite rotational direction located near the proximal (upstream) end of the bulge. The secondary vortex persists in the bulge to frame $(\mathrm{j})$, after which it is convected out of the bulge during the systolic phase of the pulse cycle.

The vorticity contour plots reveal regions of high shear along the straight tube walls, frames (a) and (c), as well as local regions of high shear at intermittent times and locations within the aneurysm bulge.

Dimensionless wall shear stress was sampled on the tube wall upstream, downstream and throughout the aneurysm bulge. These measurements are plotted in Figure 4, and demonstrate subtle variations in wall shear stress over time and as a function of position along the tube wall. In frame (a), corresponding to peak systole, a peak in the shear stress occurs in the distal end of the aneurysm $(0 \lesssim z / d \lesssim 1.45)$. This peak has a value 2.4 times greater than the level recorded in the straight portions of the tube at that time.

Over subsequent frames (b)-(c), the peak stress diminishes, but remains significantly larger than the peak value recorded in the healthy sections of the tube, occurring in frame (a). Later frames, in particular frames (d)(e), exhibit smaller stresses, sometimes almost zero throughout the tube and aneurysm. These times correspond to the resting period of the pulse cycle.

To determine how the wall shear stress measured in the aneurysm bulge differs from the levels and variation of wall shear stress in healthy vessels, time mean and standard deviations were measured over a pulse cycle at each sample location plotted in Figure 4. The resulting plots are shown in Figure 5. Significantly, deviation from the values obtained in the straight tube sections are isolated to the aneurysm bulge $(|z| / \mathrm{d} \lesssim 1.45)$. Figure $5(\mathrm{a})$ shows that within this aneurysm model, the time mean of the wall shear stress is 4.0 times greater than the mean level in the healthy vessel, and an opposite sign peak is found with a level 7.3 times greater than the healthy level. These elevated levels of wall shear stress may lead to damage of the 
(a) $t=0.0 \mathrm{~T}$

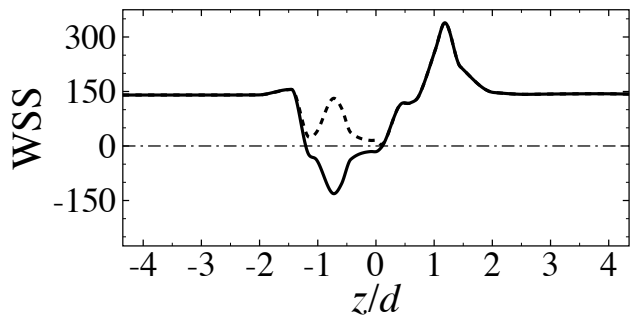

(b) $\mathrm{t}=0.2 \mathrm{~T}$

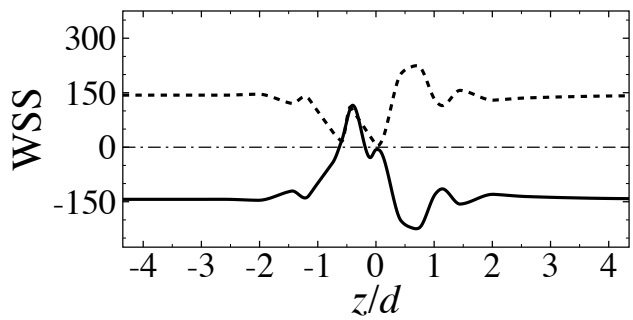

(c) $\mathrm{t}=0.4 \mathrm{~T}$

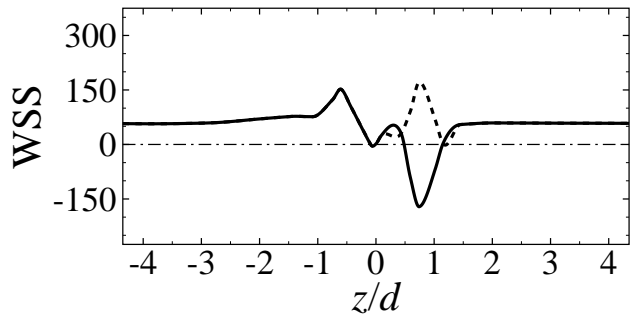

(d) $t=0.6 \mathrm{~T}$

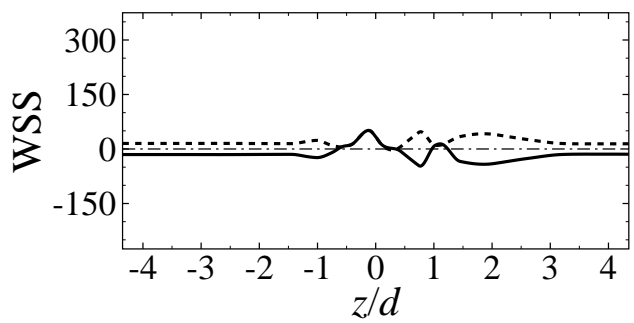

(e) $\mathrm{t}=0.8 \mathrm{~T}$

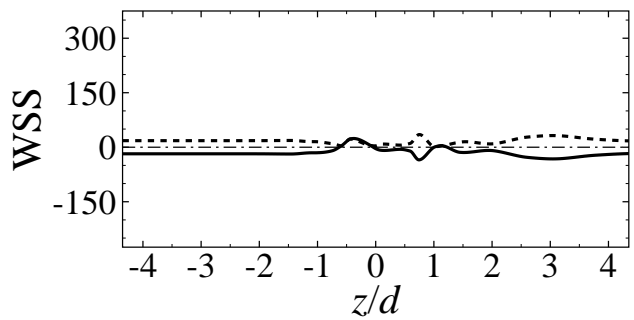

(f) $\mathrm{t}=1.0 \mathrm{~T}$

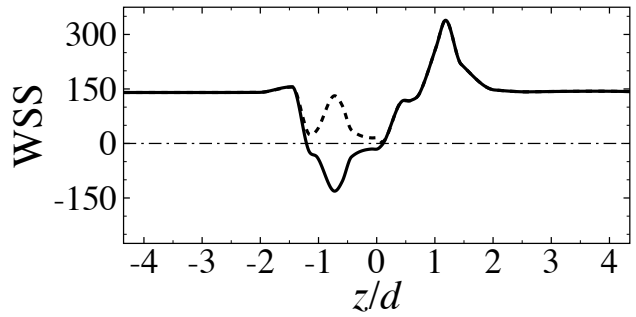

Figure 4: Plots of dimensionless wall shear stress (solid lines) and its magnitude (dotted lines) at locations along the wall upstream, within, and downstream of the aneurysm bulge. Data was acquired from the solutions shown in Figure 3. 
(a)

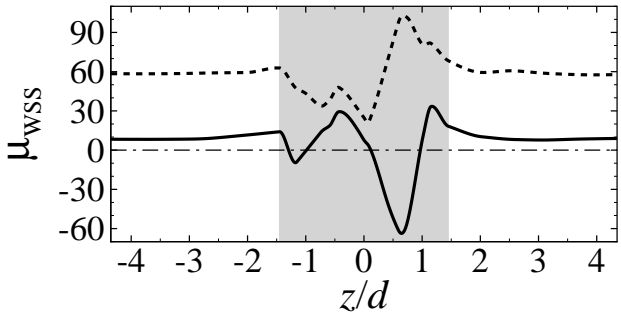

(b)

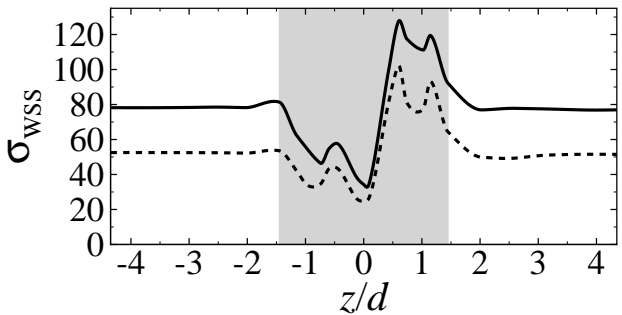

Figure 5: Plots of (a) the local time mean of both the dimensionless wall shear stress (Wss, solid line) and its magnitude (|wss $\mid$, dotted line), and (b) their standard deviations against axial position. Again, data was acquired at the conditions shown in Figure 3. The shaded region shows the location of the aneurysm bulge.

endothelium on the distal aneurysm wall [5].

The standard deviation trends in Figure 5(b) show that the magnitude of wall shear stress experiences a smaller fluctuation than the wall shear stress itself. In healthy sections of the vessel, the standard deviation for wss was $49 \%$ greater than for $|\mathrm{wsS}|$. The minimum standard deviations are found at the mid-point of the aneurysm, with values $44 \%-47 \%$ of those obtained in a healthy vessel. This could possibly degrade the integrity of the intimal layer of the vessel wall [8]. Peaks in the standard deviation were found in the distal region of the aneurysm bulge. These peaks were 194\% and 163\% of the values found in a healthy vessel for wss and $\mid$ wsS $\mid$, respectively. The experiments of Salsac et al. [13] determined wall shear stress levels within their aneurysm models to be approximately $80 \%$ of those found in healthy vessels, while conceding that those values were under predicted because of a lack of resolution. These computations demonstrate that wall shear stress levels (both in terms of a time-averaged level as well as the amount of fluctuation experienced at a given point) can differ significantly from the levels found in a healthy vessel. 
A stability analysis was employed which determined that at $R e=330$, azimuthal modes $1 \leq \mathrm{m} \leq 6$ were unstable to global non-axisymmetric instability modes, with $m=3$ being most unstable through a subharmonic bifurcation. These modes were most prominent in the distal end of the aneurysm bulge, and were flushed into the downstream tube during systole. The axisymmetric flow was found to be stable at $R e=250$, and it remains an open question as to how the development of non-axisymmetric flow affects the wall shear stress distribution.

\section{Conclusions}

Pulsatile flow has been computed in a model aneurysm defined by a sinusoidal bulge on a straight circular tube. The aneurysm dimensions $(\mathrm{LR}=2.9$, $\mathrm{DR}=1.9)$ and flow parameters $(\mathrm{Re}=330, \alpha=10.7)$ correspond to a medium sized aneurysm, typically considered low risk, on a human abdominal aorta. Significantly higher mean and standard deviation in shear stress were recorded in the distal end of the aneurysm bulge, as compared to those values recorded in straight sections of the tube. Contrary to previous experiments, these computations reveal that under these conditions the highest wall shear stress occurs at peak systole, and is 2.4 times greater than the highest wall shear stress recorded in a healthy vessel. This peak value occurred at peak systole at the distal end of the aneurysm.

Acknowledgements: G.J.S. thanks Dr. Hugh M. Blackburn for suggesting the ARPACK package for our stability analysis algorithm, and acknowledges the seed funding provided by the Monash Research for an Ageing Society (MonRAS) Research Support Scheme. The authors thank the Australian Partnership for Advanced Computing (APAC) for providing access to the computational resources of their National Facility through the Merit Allocation Scheme. 


\section{References}

[1] A. Astradsson and J. Astrup. An intracranial aneurysm in one identical twin, but no anerusym in the other. Br. J. Neurosurg., 15(2):168-171, 2001. C2

[2] D. Barkley and R. D. Henderson. Three-dimensional Floquet stability analysis of the wake of a circular cylinder. J. Fluid Mech., 322:215-241, 1996. C7

[3] H. M. Blackburn and J. M. Lopez. On three-dimensional quasi-periodic Floquet instabilities of two-dimensional bluff body wakes. Phys. Fluids, 15(8):L57-L60, 2003. doi:10.1063/1.1591771. C7

[4] H. M. Blackburn and S. J. Sherwin. Formulation of a Galerkin spectral element-Fourier method for three-dimensional incompressible flow in cylindrical geometries. J. Comput. Phys., 197:759-778, 2004. doi:10.1016/j.jcp.2004.02.013. C6

[5] D. L. Fry. Acute vascular endothelial changes associated with increased blood velocity gradients. Circ. Res., 22:165-197, 1968. C3, C11

[6] G. E. Karniadakis, M. Israeli, and S. A. Orszag. High-order splitting methods for the incompressible Navier-Stokes equations. J. Comp. Phys., 97:414-443, 1991. C6

[7] S. Kondo, N. Hashimoto, H. Kikuchi, F. Hazama, I. Nagata, and H. Kataoka. Cerebral aneurysms arising at nonbranching sites. an experimental study. Stroke, 28(2):398-403, 1997. C2

[8] D. N. Ku, D. P. Giddens, C. K. Zarins, and S. Glagov. Pulsatile flow and atherosclerosis in the human carotid bifurcation. Positive correlation between plaque location and low oscillating shear stress. Arterioscler. Thromb. Vasc. Biol., 5:293-302, 1985. C3, C11 
[9] J. C. Lasheras. The biomechanics of arterial aneurysms. Ann. Rev. Fluid Mech., 39:293-319, 2007. doi:10.1146/annurev.fluid.39.050905.110128. C2, C3

[10] R. B. Lehoucq, D. C. Sorenson, and C. Yang. ARPACK Users' Guide. SIAM: Philadelphia, PA, 1998. http://www.caam.rice.edu/software/ARPACK/UG/ug.html. C7

[11] S. Oyre, E. M. Pedersen, S. Ringgaard, P. Boesiger, and W. P. Paaske. In vivo wall shear stress measured by magnetic resonance velocity mapping in the normal human abdominal aorta. Eur. J. Vasc. Endovasc. Surg., 13(3):263-271, 1997. C3

[12] R. B. Rutherford. Randomized EVAR trials and advent of level I evidence: A paradigm shift in management of large abdominal aortic aneurysms? Semin. Vasc. Surg., 19(2):69-74, 2006. doi:10.1053/j.semvascsurg.2006.03.001. C2

[13] A.-V. Salsac, S. R. Sparks, J.-M. Chomaz, and J. C. Lasheras. Evolution of the wall shear stresses during the progressive enlargement of symmetric abdominal aortic aneurysm. J. Fluid Mech., 560:19-51, 2006. doi:10.1017/S00221120060036X. C3, C4, C5, C11

[14] G. J. Sheard and K. Ryan. Pressure-driven flow past spheres moving in a circular tube. J. Fluid Mech., 592:233-262, 2007. doi:10.1017/S0022112007008543. C6

[15] T. B. M. Wilmink, C. R. G. Quick, and N. E. Day. The association between cigarette smoking and abdominal aortic aneurysms. J. Vasc. Surg., 30:1099-1105, 1999. C2

[16] J. R. Womersley. Method for the calculation of velocity, rate of flow and viscous drag in arteries when the pressure gradient is known. J. Physiol., 127:553-563, 1955. C7 


\section{Author addresses}

1. G. J. Sheard, Monash University Biomedical Engineering Technology Alliance, Division of Biological Engineering, Faculty of Engineering, Monash University, Victoria 3800, AUstralia. mailto:Greg. Sheard@eng. monash. edu .au

2. K. Ryan, Fluids Laboratory for Aeronautical and Industrial Research, Department of Mechanical and Aerospace Engineering, Monash University, Victoria 3800, Australia.

mailto:Kris.Ryan@eng.monash. edu.au 